\title{
Arginase isoenzymes in human cirrhotic liver
}

\author{
Alicja Chrzanowska, Beata Gajewska and Anna Barańczyk-Kuźma ${ }^{\bowtie}$
}

Chair and Department of Biochemistry, Medical University of Warsaw, Warszawa, Poland

Received: 20 May, 2009; revised: 30 June, 2009; accepted: 20 July, 2009

available on-line: 27 July, 2009

\begin{abstract}
Cirrhosis leads to an inability of the liver to perform its biochemical functions. It can also lead to hepatocellular carcinoma in which, as we showed lately, arginase isoenzyme pattern changes. The present work presents our results on arginase isoenzymes and their possible role in liver cirrhosis. The study was performed on tissues obtained during liver transplantation from 60 patients with liver cirrhosis, and on samples of histologically normal liver (control) from 40 patients with benign or colorectal cancer liver metastases removed during surgery, $6-7 \mathrm{~cm}$ from the tumor border. Arginase isoenzymes AI (so-called liver-type arginase) and AII (called extrahepatic arginase) were identified by Western blotting and isolated by ion-exchange chromatography. Their expression on mRNA level was studied by RT-PCR. A significant decrease in arginase activity, dependent of the liver clinical stage, was observed in cirrhotic tissue. Arginase AI activity and its mRNA level were significantly decreased in cirrhotic liver, whereas the activity and expression of arginase AII were concurrently raised, as compared to normal liver. Since arginase AI is a key enzyme of the urea cycle, whereas arginase AII most probably takes part in the biosynthesis of ornithine and polyamines, the defective ammonia inactivation and increased collagen biosynthesis observed in cirrhotic liver may be related to the changes in arginase AI and AII levels, respectively.
\end{abstract}

Keywords: arginase activity, isoenzyme expression, liver cirrhosis

\section{INTRODUCTION}

The liver is involved in nearly all aspects of metabolism. It is also the main organ responsible for enzymatic detoxification of both exo- and endogenic compounds. As a consequence, many harmful substances get into the liver or are produced inside hepatocytes. When not inactivated properly, they can cause liver damage. Liver cells may then regenerate in an abnormal pattern forming nodules that are surrounded by fibrous tissue. Cirrhosis is characterized anatomically by widespread nodules combined with fibrosis (Hillaire \& Voitot, 1999). The main risk factors for liver cirrhosis are alcoholism and chronic hepatitis B or C virus infections (Aguilera et al., 2009). Some patients with liver cirrhosis, especially early in the course of the disease, have no clinical signs or symptoms. Biochemical and radiological tests are used for the diagnosis. However, in some cases the diagnosis must be confirmed by liver biopsy (Dioguardi et al., 2008). Liver cirrhosis is irreversible but treatment of underlying liver diseases may slow or even stop its progression. Liver transplantation is highly effective for the treatment of end-stage cirrhosis (Ferraz-Neto et al., 2007). Serious hepatocellular dysfunctions lead to disturbances in ammonia metabolism and increase the risk for hepatic encephalopathy (Kato et al., 2008).

The main process in ammonia detoxification is the urea cycle, which is localized mainly in the liver. The last enzyme of the cycle is arginase (EC 3.5.3.1) that hydrolyzes L-arginine to urea and L-ornithine. There are two arginase isoenzymes encoded by distinct genes in mammalian tissues, called arginase AI (cytosolic isoform) and AII (mitochondrial isoform) (Sparkes et al., 1986; Vockley et al., 1996). Arginase AI has the highest activity in the liver however, it occurs also in other tissues. Arginase AII is present

Corresponding author: Anna Barańczyk-Kuźma, Chair and Department of Biochemistry, Medical University of Warsaw, St. Banacha 1, 02-097 Warszawa, Poland; phone: (48) 22572 0693; fax: (48) 22572 0679; e-mail: anna.kuzma@wum.edu.pl Abbreviations: CRCLM, colorectal cancer liver metastases; HCC, hepatocellular carcinoma; RT-PCR, reverse transcriptase-polymerase chain reaction. 
in various tissues such as kidney, small intestine, stomach, pancreas, brain, and also in liver. In human liver arginase AI (previously called "hepatic arginase") is a key enzyme of the urea cycle and occurs in periportal hepatocytes, whereas arginase AII (so called "extrahepatic arginase") is localized in perivenous hepatocytes and its function is still poorly understood (O'Sullivan et al., 1998; Nissim et al., 2005). Besides urea, arginase takes part in the biosynthesis of ornithine - a parent compound for proline (for collagen), glutamate (for glutamine) and polyamines (for regulation of cell proliferation and regeneration). Arginase also competes with nitric oxide synthase for the common substrate, arginine (Cederbaum et al., 2004). Being involved in so many important biochemical pathways, arginase may be a key point in the development of various pathological processes.

In our previous work we showed changes in arginase isoenzyme pattern in hepatocellular carcinoma (HCC) and a significant decrease of arginase activity in tumor tissue, when compared with normal liver (Chrzanowska et al., 2008). We also observed a decrease of arginase activity in cirrhotic liver, used as a second control. Since about $80 \%$ of hepatocellular carcinomas develop in cirrhotic liver, in the present work we concentrated our study on arginase isoenzymes in human cirrhotic liver.

\section{MATERIALS AND METHODS}

Patients and tissues. The study was performed on cirrhotic liver obtained during liver transplantation from 60 patients with liver cirrhosis, and on histologically normal liver (control) from 40 patients with benign or colorectal cancer liver metastases (CRCLM), removed during surgery, $6-7 \mathrm{~cm}$ from the tumor border. The causes of liver cirrhosis were hepatitis B or C, alcoholism, and in single cases primary biliary cirrhosis and autoimmune hepatitis. The patients were treated in the Department of General, Transplant and Liver Surgery, and in the Department of General and Transplantation Surgery, Transplantation Institute, Medical University of Warsaw. The group with liver cirrhosis comprises 24 females and 36 males, aged 47.0 \pm 8.4 (range 19-63 years), and the control group 22 females and 18 males, aged $56.4 \pm 9.8$ (range 32-76 years). The patients with liver cirrhosis were diagnosed by blood liver tests, ultrasound sonography, computed tomography and the level of carcinoembrionic antigen (for the patients with colorectal cancer liver metastases). The efficiency of liver function was estimated according to the ChildPugh score (Huo et al., 2005). The studies were approved by the Bioethics Committee of the Medical
University of Warsaw, and informed consent was obtained from all patients.

Methods. Immediately after surgical removal, the resected tissues were washed in $0.9 \% \mathrm{NaCl}$ and frozen at $-80^{\circ} \mathrm{C}$. The tissues were homogenized in 10 vol. of cold $50 \mathrm{mM}$ Tris/ $\mathrm{HCl}$ buffer, $\mathrm{pH} 7.5$, containing $1 \mathrm{mM} \mathrm{MnCl}, 0.2 \mathrm{M} \mathrm{KCl}$, and $0.1 \%(\mathrm{v} / \mathrm{v})$ Triton X-100 using a Heidolph Diax 900 blender at low speed, 5 times for $2 \mathrm{~min}$ at $3 \mathrm{~min}$ intervals. After 30 min extraction on a magnetic stirrer, the homogenates were centrifuged at $12000 \times \mathrm{g}$ for $30 \mathrm{~min}$ at $4^{\circ} \mathrm{C}$. The supernatants were used for arginase activity determination, isolation of isoenzymes by ion-exchange chromatography, and Western blotting analysis.

Arginase activity was measured by determining the increase in the amount of ornithine (Chinard, 1952). One unit (U) of enzymatic activity was defined as that forming $1 \mu \mathrm{mol}$ of the product per minute at $37^{\circ} \mathrm{C}$, and was expressed per $1 \mathrm{~g}$ of wet tissue.

Western blotting was performed after electrophoresis in $14 \%$ polyacrylamide gel (Laemmli, 1970), with rabbit anti-bovine arginase AI and AII polyclonal antibodies (Santa Cruz Biotech. Inc., USA). Arginase AI from bovine liver and AII from human kidney were used as standards. Blots were visualized using ECL plus Western Blotting Detection System (Amersham).

Ion-exchange chromatography was performed on a CM-cellulose column $(10 \times 1.5 \mathrm{~cm})$ equilibrated with $20 \mathrm{mM}$ Tris/ $\mathrm{HCl}$ buffer, 7.5. The non-adsorbed arginase AII (anionic isoform) was eluted with the same buffer, and the adsorbed arginase AI (cationic isoform) with a linear $\mathrm{KCl}$ gradient $(0.5-1.0 \mathrm{mM})$. The active fractions were pooled and the total activity of either arginase form was established.

Total RNA was isolated using TRIzol ${ }^{\circledR}$ reagent (Invitrogen) according to manufacturer's protocol (Chomczynski \& Sacchi, 1987). Expression levels of AI-mRNA and AII-mRNA were defined by reverse transcriptase-polymerase chain reaction (RT-PCR). Specific oligonucleotide primers for arginase isoenzymes were based on nucleotide sequences in NCBI accession No. NM000045 for ARGI and BC029050 for ARGII, and were as follows: for arginase AI: TGCAACTGCTGTGTTCACTG (forward), TGATGTTGACGGACTGGACC (reverse), for arginase AII: GTTAGCAGAGCTGTGTCAGA (forward), GGAGTTGTGGTACCTTATCC (reverse). The specific mRNA sequence for $\beta_{2}$-microglobulin (a housekeeping gene) was amplified as described by Brophy et al. (1994), and served as an internal control (forward primer: CCAGCAGAGAATGGAAAGTC, reverse primer: GATGCTGCTTACATGTCTCG). PCR products were separated on $1.5 \%$ agarose gel with ethidium bromide. The level of specific mRNA was measured and expressed as the ratio of optical density band of arginase AI and arginase AII to optical density 
band of $\beta_{2}$-microglobulin. Each assay was repeated two times and performed in duplicate. UVI-KS4000 system (Syngen Biotech.) was used for densitometric analysis of Western blotting and RT-PCR results.

Results were expressed as means \pm S.D. Quantitative comparison between studied groups was performed by Student's $t$-test using Statistica software (StatSoft 8.0) and non-parametric Mann-Whitney $\mathrm{U}$ test.

\section{RESULTS}

Arginase activity in cirrhotic livers ranged from 41.8 to $888.5 \mathrm{U} / \mathrm{g}$ of wet tissue. The mean activity was $462.0 \pm 210.8 \mathrm{U} / \mathrm{g}$, and it was significantly lower than in control livers, were it ranged from 860.4 to $2300.0 \mathrm{U} / \mathrm{g}$, with the mean of $1305.8 \pm 515.2$ $\mathrm{U} / \mathrm{g}$ of wet tissue. The activity in cirrhotic liver was dependent of the liver clinical stage as expressed in the Child-Pugh score (Fig. 1). Activity in stage A was $791.0 \pm 130.3$, in stage $B-473.1 \pm 148.3$, and in stage $C-121.1 \pm 57.2 \mathrm{U} / \mathrm{g}$ of wet tissue. The differences between patient groups and control, between groups $\mathrm{B}$ and $\mathrm{A}$, and groups $\mathrm{C}$ and $\mathrm{B}$ were all statistically significant $(P<0.001)$.

Two arginase isoenzymes - AI and AII were identified by Western blotting in cirrhotic liver (Fig. 2A and B). When separated by ion-exchange chromatography AI accounted for about $80 \%$ and AII $20 \%$ of the total arginase activity, whereas in the control liver - about 90\% (AI) and 10\% (AII).

Arginase AI mRNA expression in cirrhotic liver was $1.07 \pm 0.06$ (range from 0.97 to 1.19 ), whereas in the control tissue it was $1.38 \pm 0.1$ (range from 1.22 to 1.56 ). The mean value of AII mRNA expres-

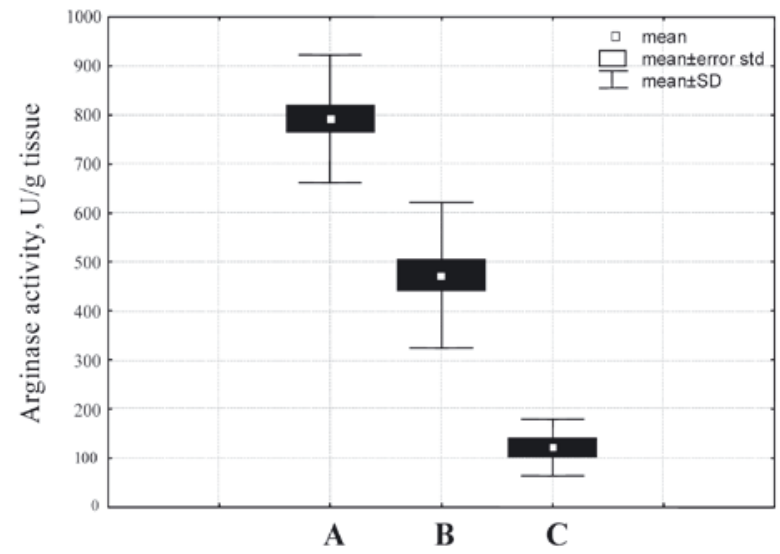

Figure 1. Dependence of arginase activity on clinical stage of liver cirrhosis.

Activity was determined as indicated in Materials and Methods. Liver cirrhosis was estimated according to Child-Pugh classification from A to $\mathrm{C}$, with $\mathrm{C}$ indicating the most severe derangement. Group A contained 21 patients, group $B-20$ patients, and group $C-19$ patients.

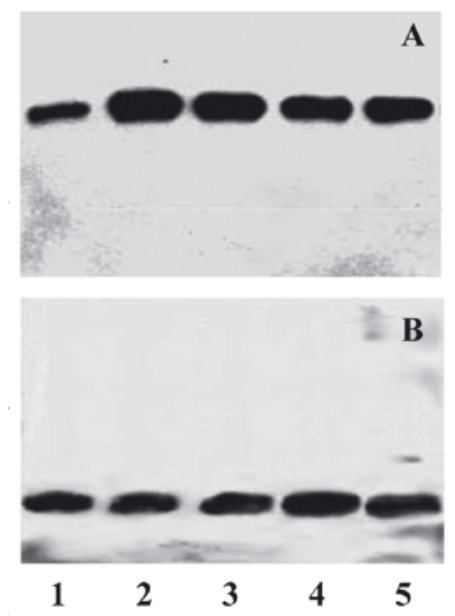

Figure 2. Western blot analysis of arginase isoenzyme AI (A) and AII (B) in cirrhotic liver.

Comparable amounts of protein $(5 \mu \mathrm{g})$ were run in each lane, as described in Materials and Methods. A: lane 1, standard bovine liver arginase AI; lanes 2 and 3, arginase AI from control liver removed $6-7 \mathrm{~cm}$ from the border of benign tumor and CRCLM, respectively; lanes 4 and 5, arginase AI from cirrhotic liver. B: lane 1, standard human kidney arginase AII; lanes 2 and 3, arginase AII from control liver removed $6-7 \mathrm{~cm}$ from border of benign tumor and CRCLM, respectively; lanes 4 and 5, arginase AII from cirrhotic liver.

sion was $0.27 \pm 0.05$ in cirrhotic liver (range from 0.20 to 0.35 ), and in the control tissue it was $0.12 \pm 0.04$ (range from 0.09 to 0.19 ). The decrease of AI mRNA and the increase of AII mRNA level in cirrhotic liver were statistically significant $(P<0.001)$ when compared with the control (Figs. 3 and 4 ).

\section{DISCUSSION}

Hepatocytes are homogenous in structure but differ in metabolic activity. Two efficient enzymatic

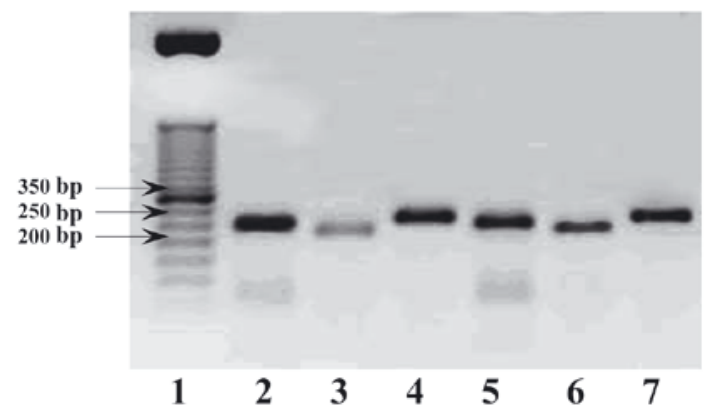

Figure 3. Expression of AI mRNA and AII mRNA in cirrhotic liver.

RT-PCR was performed as described in Materials and Methods. Similar amount $(12 \mu \mathrm{l})$ of PCR product was run in each lane. Lane 1, ladder (base pairs); lanes 2, 3, 4, AI, AII, $\beta_{2}$-microglobulin from control liver, respectively; lanes 5, 6, 7, AI, AII, $\beta 2$-microglobulin from cirrhotic liver, respectively. 


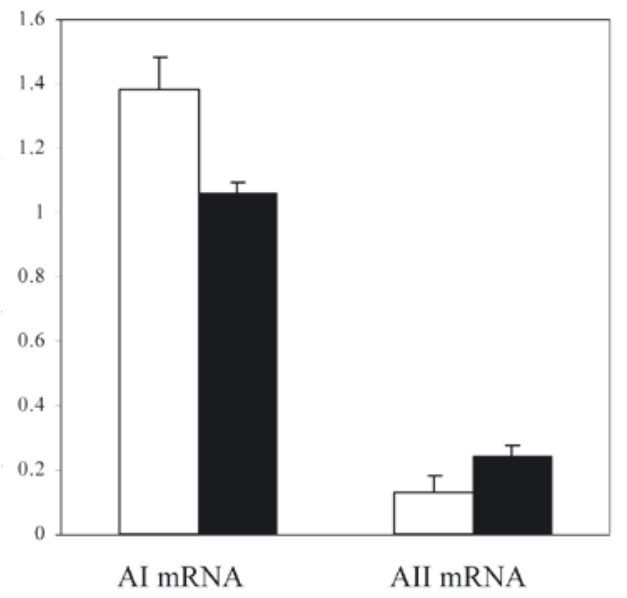

Figure 4. Comparison of arginase isoenzyme expression in control and cirrhotic liver.

Open bars: AI mRNA and AII mRNA in control liver; solid bars: AI mRNA and AII mRNA in cirrhotic liver. Bars represent the means \pm S.D.

processes, the urea cycle and glutamine biosynthesis, are involved in ammonia detoxification in the human liver. All urea cycle enzymes are localized in periportal hepatocytes, whereas glutamine synthetase occurs in perivenous hepatocytes (Häussinger et al., 1992; Gebhardt et al., 2007). It is known that the arginase isoenzymes present in human liver differ in their acinar localization and physiological function (Wheatley \& Campbell, 2002). Arginase AI occurs in the periportal zone and participates in ammonia detoxification, whereas arginase AII is localized in perivenous hepatocytes and utill now its function is not clear. It can supply cells with ornithine, a precursor of polyamines and a substrate for proline and glutamate synthesis (Cederbaum et al., 2004). Since liver is the main organ responsible for inactivation of various xenobiotics, it is particulary exposed to their toxic effects. One of the most common liver disorders is cirrhosis, which in most cases can lead to the development of hepatocellular carcinoma. The development of liver cirrhosis is difficult to diagnose since the symptoms are not characteristic and they usually appear late in the course of the disease (Hillaire \& Voitot, 1999; Shah et al., 2006).

In our previous study, focused on hepatocellular carcinoma, we found that arginase activity in the cancerous tissue of HCC was 15-fold lower, whereas in the cirrhotic liver (used as one of the controls) it was nearly 3 -fold lower than in normal liver (Chrzanowska et al., 2008). In the present study performed on a higher number of cirrhotic cases, we confirmed the 3-fold decrease of arginase activity. The analysis of activity showed that the decrease was associated with impaired cirrhotic liver function estimated according to the Child-Pugh classification. At stage A the activity was about 2-fold lower, at stage $\mathrm{B}$ about 3-fold lower, and at stage $\mathrm{C}$ above 10fold lower than in control. The dependence of arginase activity on the clinical stage of liver cirrhosis is most probably related to the loss of hepatocytes and the concurrent development of fibrous tissues. Considering that arginase is the crucial enzyme for the urea cycle, a decrease of its activity can lead to disturbances in ammonia detoxification followed by hyperammonemia.

Similarly as in normal liver and in HCC, in cirrhotic liver we observed the presence of isoenzymes AI and AII. Arginase AI accounted for about $80 \%$ of the total activity, which was lower than in normal liver (at least 90\%), but higher than in HCC (about 60\%) (Chrzanowska et al., 2008). The decrease of arginase AI activity was accompanied by an increase of AII, the activity of which was 2-fold higher in cirrhotic liver than in control tissue. The changes in the activity reflected expression of the arginase isoenzymes on the level of mRNA. The expression of AI mRNA was lower in cirrhotic than in control liver (77.5\%), whereas the expression of AII mRNA was higher $(225 \%)$. Such changes in both expression and activity of the arginase isoenzymes may allow compensation of ammonia detoxification in various zones of hepatic acinus in cirrhotic liver. The decrease of arginase AI may impair the urea cycle in the periportal zone, whereas the rise of arginase AII may result in activation of glutamine synthesis (from glutamate) in the perivenous zone. Numerous studies indicate the induction of arginase AII in patients with urea cycle disorders caused by arginase AI deficiency (Grody et al., 1993; Cederbaum et al., 2004; Crombez \& Cederbaum, 2005). The raised arginase AII may be useful for regeneration of damaged hepatocytes, since it can deliver ornithine for biosynthesis of polyamines. However, the intensified synthesis of collagen in cirrhotic liver consequently rises the demand for proline, which is derived from ornithine synthesized by arginase AII. Thus, it is possible that arginase AII facilitates development of fibrosis. Studies on fibrosis in mouse lungs have demonstrated induction of arginase AII in myofibroblasts (Endo et al., 2003). A similar process may occur in myofibroblasts that are abundantly present in cirrhotic liver. Fibrosis is critical for the development of cirrhosis and although numerous studies have been undertaken regarding its inhibition in cirrhotic liver, but there is still no effective method to prevent it (Olga \& Nikolai, 2003; Yu et al., 2009). Arginase AII may be involved in this process, but more studies are needed to find out its precise role.

\section{Acknowledgements}

The authors wish to thank Professor Marek Krawczyk from the Department of General, Transplant and Liver Surgery, and Professor Zbigniew 
Wierzbicki from the Department of General and Transplantation Surgery, Transplantation Institute, Medical University of Warsaw (Warszawa, Poland) for their help in obtaining human tissues.

This study was supported by grant $1 \mathrm{WK} /$ WB1/08 from the Medical University of Warsaw (Warszawa, Poland).

\section{REFERENCES}

Aguilera V, Berenguer M, Rubin A, San-Juan F, Rayón JM, Prieto M, Mir J (2009) Cirrhosis of mixed etiology (hepatitis $\mathrm{C}$ virus and alcohol): Posttransplantation outcome - Comparison with hepatitis C virus-related cirrhosis and alcoholic-related cirrhosis. Liver Transpl 15: 79-87.

Brophy NA, Marie JP, Rojas VA, Warnke RA, McFall PJ, Smith SD, Sikic BI (1994) Mdr1 gene expression in childhood acute lymphoblastic leukemias and lymphomas: a critical evaluation by four techniques. Leukemia 8: $327-335$.

Chinard FP (1952) Photometric estimation of proline and ornithine. J Biol Chem 199: 91-95.

Chomczynski P, Sacchi N (1987) Single-step method of RNA isolation by acid guanidium thiocyanate-phenolchloroform extraction. Anal Biochem 162: 156-159.

Chrzanowska A, Krawczyk M, Barańczyk-Kuźma A (2008) Changes in arginase isoenzymes pattern in human hepatocellular carcinoma. Biochem Biophys Res Commun 377: 337-340.

Cederbaum SD, Yu H, Grody WW, Kern RM, Yoo P, Iyer RK (2004) Arginases I and II: do their functions overlap? Mol Genet Metab 8: S38-S44.

Crombez EA, Cederbaum SD (2005) Hyperargininemia due to liver arginase deficiency. Mol Genet Metab 84: 243-2451.

Dioguardi N, Grizzi F, Fiamengo B, Russo C (2008) Metrically measuring liver biopsy: A chronic hepatitis B and C computer-aided morphologic description. World J Gastroenterol 14: 7335-7344.

Endo M, Oyadomari S, Terasaki Y, Takeya M, Suga M, Mori M, Gotoh T (2003) Induction of arginase I and II in bleomycin-induced fibrosis of mouse lung. Am J Physiol Lung Cell Mol Physiol 285: 313-321.

Ferraz-Neto BH, Hidalgo R, Thomé T, Melo VA Jr, Lobue A, Zurstrassen MP, Moraes JM Jr, Meira-Filho SP, Rezende MB, Fonseca LE, Pandullo FL, Soeiro FS, Afonso RC (2007) Analysis of model for end-stage liver disease (MELD) score in a liver transplantation waiting list. Transplant Proc 8: 2511-2513.

Gebhardt R, Baldysiak-Figiel A, Krügel V, Ueberham E, Gaunitz F (2007) Hepatocellular expression of glutamine synthetase: an indicator of morphogen actions as master regulators of zonation in adult liver. Prog Histochem Cytochem 41: 201-266.

Grody WW, Kern RM, Klein D, Dodson AE, Wissman PB, Barsky SH, Cederbaum SD (1993) Arginase deficiency manifesting delayed clinical sequelae and induction of kidney arginase isoenzyme. Hum Genet 91: 1-5.

Häussinger D, Lamers WH, Moorman AF (1992) Hepatocyte heterogenity in the metabolism of amino acids and ammonia. Enzyme 46: 72-93.

Hillaire S, Voitot H (1999) Cirrhosis. Pathol Biol 47: 895905.

Huo TI, Wu JC, Lin HC, Lee FY, Hou MC, Lee PC, Chang FY, Lee SD (2005) Evaluation of the increase in model for end-stage liver disease (DeltaMELD) score over time as a prognostic predictor in patients with advanced cirrhosis: risk factor analysis and comparison with initial MELD and Child-Turcotte-Pugh score. J Hepatol 42: 826-832.

Kato A, Watanabe Y, Sawara K, Suzuki K (2008) Diagnosis of sub-clinical hepatic encephalopathy by neuropsychological tests (NP-tests). Hepatol Res 38: 122-127.

Kern RM, Grody WW, Cederbaum SD, Kern RM, Yoo P, Iyer RK Laemmli UK (1970) Cleavage of structural proteins during the assembly of the head of bacteriophage T4. Nature 227: 680-685.

Nissim I, Luhovyy B, Horyn O (2005) The role of mitochondrially bound arginase in regulation of urea synthesis. J Biol Chem 280: 17715-17724.

Olga OZ, Nikolai DY (2003) Invasive and non-invasive monitoring of hepatitis $C$ virus-induced liver fibrosis: alternatives or complements? Curr Pharm Biotechnol 4: 195-209.

O'Sullivan D, Brosnan JT, Brosnan ME (1998) Hepatic zonation of the catabolism of arginine and ornithine in the perfused rat liver. Biochem J 330 (Part 2): 627-632.

Shah TU, Semelka RC, Pamuklar EZ, Firat Z, Gerber RD, Shrestha R, Russo MW (2006) The risk of hepatocellular carcinoma in cirrhotic patients with small liver nodules on MRI. Am J Gastroenterol 101: 533-540.

Sparkes RS, Dizikes GJ, Klisak I, Grody WW, Mohandas T, Heinzmann C, Zollman S, Lusis AJ, Cederbaum SD (1986) The gene for human liver arginase (ARG1) is assigned to chromosome band 6q23. Am J Hum Genet 39: 186-191.

Vockley JG, Jenkinson CP, Shukala H, Kern RM, Grody WW, Cederbaum SD (1996) Cloning and characterization of the human type II arginase gene. Genomics 38: 118-123.

Wheatley DN, Campbell E (2002) Arginine catabolism, liver extracts and cancer. Pathol Oncol Res 8: 18-25.

Yu J, Hui AY, Chu ES, Go MY, Cheung KF, Wu CW, Chan HL, Sung JJ (2009) The anti-inflammatory effect of celecoxib does not prevent liver fibrosis in bile duct-ligated rats. Liver Int 29: 25-36. 